SCHNEIDER, G.

\title{
Bifurcation Theory for Dissipative Systems on Unbounded Cylindrical Domains - An Introduction to the Mathematical Theory of Modulation Equations -
}

\begin{abstract}
Lecture presented at the 77th Annual GAMM Conference, Metz, April 12-16, 1999
In this introductory text we consider dissipative translational invariant nonlinear partial differential equations posed on spatially unbounded cylindrical domains close to the threshold of instability. We give an overview about the mathematical theory of modulation equations which allows us to analyse bifurcation problems occurring in such systems. We explain this theory by presenting new results concerning the dynamics of interface solutions bifurcating in certain reaction-diffusion systems.
\end{abstract}

Key words: reduction, interfaces, Allen-Cahn equation

MSC (2000): 35A35, 35K57, 35Q60, 34A99

\section{Introduction}

In many cases the scientific understanding of nature is highly related to the understanding of the underlying nonlinear partial differential equations. The use of computers has given new insight into the behavior of these infinite-dimensional dynamical systems, and so in the last years nonlinear systems play a big role in modern science. On the numerical side big efforts are made in the construction of algorithms for the efficient simulation of such systems [63].

A very successful method to understand dissipative nonlinear systems analytically is based on bifurcation theory $[8,22-24]$. The idea is to analyse the instabilities of the system and to follow the bifurcating solutions in parameter space in order to gain an almost complete picture of the behavior of the nonlinear system. For bounded domains center manifold theory and the Lyapunov-Schmidt method are powerful tools to reduce a priori infinite dimensional bifurcation problems to finite dimensional ones.

Here we are interested in systems, where such a reduction is not possible, namely dissipative translational invariant nonlinear partial differential equations posed on spatially unbounded cylindrical domains close to the threshold of instability. Such systems are an idealisation of spatially extended systems. A system is called spatially extended if i) the internal wavelength of the system or ii) the size of a spatially localised structure is much smaller than the size of the underlying physical domain, for instance i) the distance between two sand ripples on a beach or dune or ii) the center of a tornado. If we measure the applicability of the center manifold theorem or of the Lyapunov-Schmidt method in terms of the possible size of the bifurcation parameter, this applicability goes to zero, as the length of the domain goes to infinity.

Estimates for the approximation of partial differential equations posed on spatially extended, but bounded domains by those posed on unbounded domains show that the error made by this idealisation grows, as usual, exponentially in time, but decays exponentially with the distance from the boundaries [43]. Thus, the consideration of partial differential equations posed on spatially unbounded cylindrical domains is a good approximation of reality and not an academical artifact.

It is the aim of this introductory paper to give a survey of new developments in bifurcation theory for problems posed on spatially unbounded cylindrical domains $\mathbb{R} \times \Sigma$, with $\Sigma \subset \mathbb{R}^{d}$ a bounded smooth domain. Due to the negligence of far away boundaries Fourier transform is available in the unbounded direction(s) and so called modulation or envelope equations can be derived as formal approximation equations [13, 45]. Examples for such simple partial differential equations are the KPP-equation, the Ginzburg-Landau equation, or the Cahn-Hilliard equation. They take the role of the finite-dimensional amplitude equations on the center manifolds used in classical bifurcation theory. In the last years great efforts are made to develop a mathematical theory for the description of bifurcation problems posed on spatially unbounded cylindrical domains by these modulation or envelope equations (cf. [11, 15, 48, 66]). In [59] it was pointed out that the mathematical theory of modulation equations for bifurcation problems posed on spatially unbounded cylindrical domains can now be considered as a generalisation of the center manifold theorem.

The plan of this overview is as follows. Sections 2 and 4 are mainly devoted to bifurcation problems posed on bounded domains. We classify the generic instabilities and bifurcations and explain why these instabilities and bifurcations occur for many completely different systems. In the same spirit in Sections 3 and 5 we classify the generic instabilities and possible modulation equations for bifurcation problems posed on spatially unbounded domains. In Section 6 the mathematical theory of modulation equations is explained by presenting new results concerning the dynamics of interface solutions bifurcating in certain reaction-diffusion systems.

Throughout this paper many constants are uniformly denoted with $C$. 


\section{Linear stability analysis for bounded domains}

In this section we explain that for problems posed on spatially bounded domains there are generically two possibilities how instability of a fixed point can occur. equation

Stability by linearisation is one basic concept of stability theory. As an example consider the ordinary differential

$$
\dot{u}=A u+f(u)
$$

with $u \in \mathbb{R}^{d}, A \in \mathbb{R}^{d \times d},\|f(u)\|_{\mathbb{R}^{d}} \leq C\|u\|_{\mathbb{R}^{d}}^{2}$ for small $u$, and $A$ depending on some parameter $\alpha \in \mathbb{R}$. The fixed point $u=0$ is called stable, if for all $\varepsilon>0$ there exists a $\delta>0$ such that solutions $u=u(t)$ with $\|u(0)\|_{\mathbb{R}^{d}} \leq \delta$ satisfy $\sup \|u(t)\|_{\mathbb{R}^{d}} \leq \varepsilon$, i.e., solutions to small perturbations stay close to $u=0$ for all times. It is well known (cf. [9]) that $t \geq 0$

stability of $u=0$ follows if the eigenvalues $\lambda_{j}$ with associated eigenvectors $\varphi_{j}$ of the linearisation $A$ have strictly negative real part. In this case additionally the asymptotic stability of the fixed point $u=0$, i.e. $\lim _{t \rightarrow \infty} u(t)=0$, holds. Solutions $u=u(t)$ to initial conditions $u(0)$ satisfying $\|u(0)\|_{\mathbb{R}^{d}}<\delta$ converge with some rate $\mathrm{e}^{-\beta t}$ to $u=0$, where $\beta<-\sup \operatorname{Re} \lambda_{j}$. $j=1, \ldots, d$
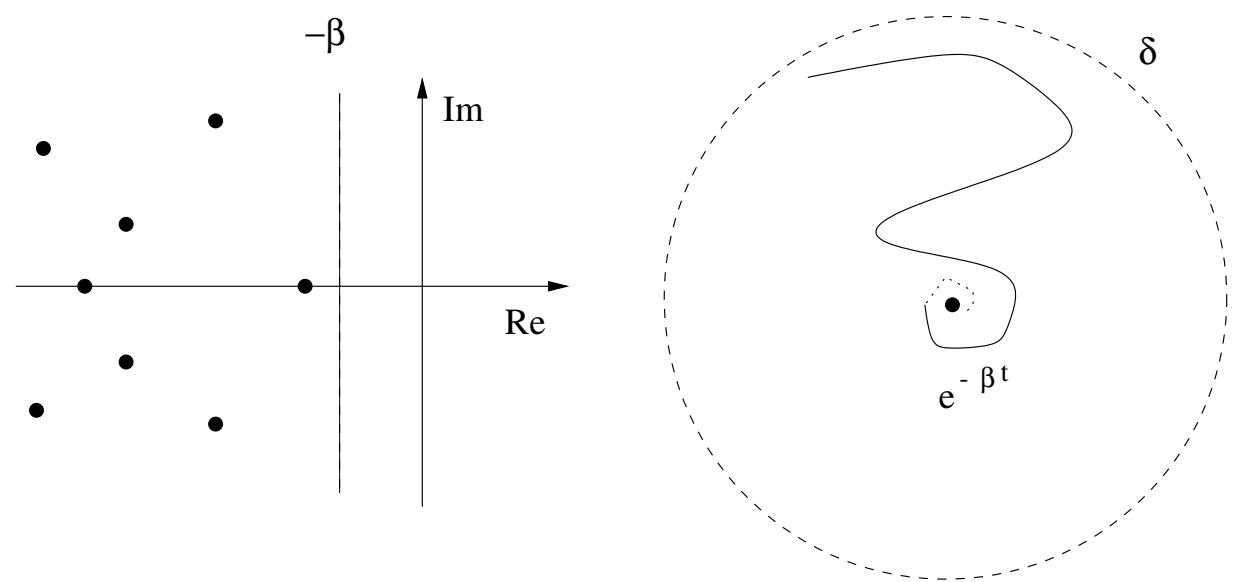

Fig. 1. Eigenvalues of $A$ determine the stability

So in a whole neighborhood of $u=0$ there are no nontrivial solutions $\left(\limsup _{t \rightarrow \infty} u(t) \neq 0\right)$. Interesting dynamics can only occur if $u=0$ changes stability. Let us assume that all eigenvalues of $A$ have strictly negative real part for $\alpha<\alpha_{c}$ and that $u=0$ changes stability at $\alpha=\alpha_{c}$. There are two generic ways in a real-valued problem how such an instability of $u=0$ can occur (cf. Fig. 2).

In Section 4 we will see that case a) will lead to a bifurcation of fixed points. Case b) will lead to a bifurcation of time-periodic solutions, i.e. to a so called Hopf-bifurcation [38].

Bifurcations a) and b) occur very often since the probability, that two real eigenvalues, or a real and a pair of complex conjugate eigenvalues, etc., cross the imaginary axis for the same value of $\alpha$, vanishes.

This situation can be transfered almost one to one to dissipative systems given by nonlinear partial differential equations, where the spatial variable lives in a bounded domain $\Sigma \subset \mathbb{R}^{d}$. Consider again (1), but now with $u \in X, X$ some Banach space, $A$ some sectorial operator in $X$ with domain of definition $X^{1}$, and $\|f(u)\|_{X} \leq C\|u\|_{X^{\alpha}}^{2}$ for small $u$,

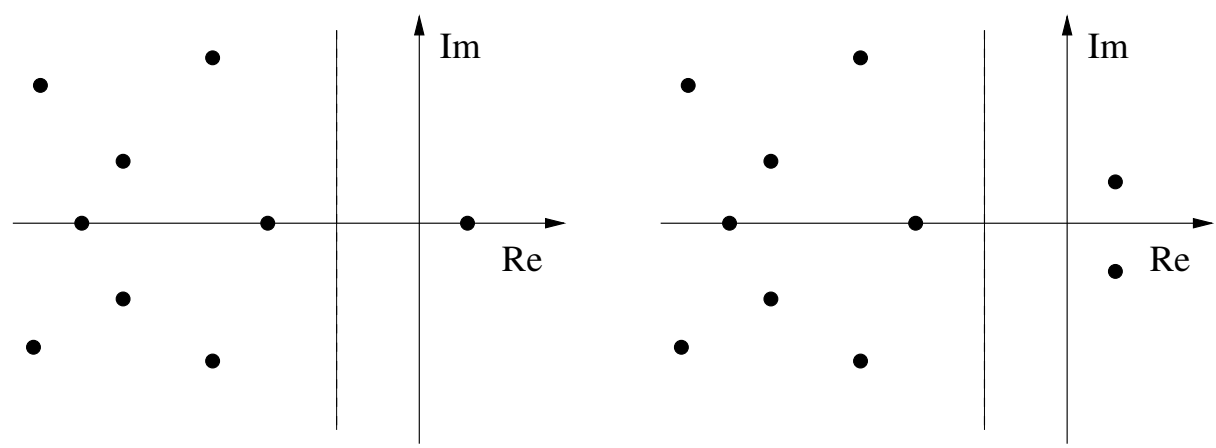

Fig. 2. a) One single real eigenvalue crosses the imaginary axis. b) A pair of complex conjugate eigenvalues crosses the imaginary axis. All other eigenvalues have strictly negative real part 

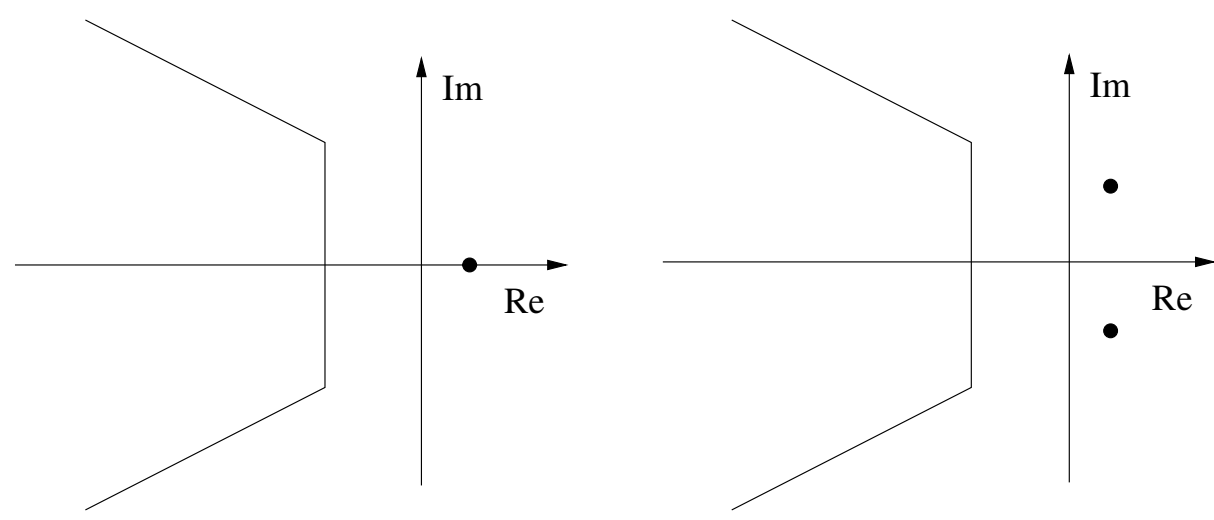

Fig. 3. a) One single real eigenvalue crosses the imaginary axis. b) A pair of complex conjugate eigenvalues crosses the imaginary axis. All other eigenvalues contained in some sector have strictly negative real part

where $X^{\alpha}$ is some interpolation space in between $X$ and $X^{1}$ (cf. [26]). If $(A-\lambda)^{-1}$ is some compact operator for some $\lambda$, the operator $A$ has discrete spectrum consisting only of eigenvalues with no point of accumulation. Since by definition the spectrum of a sectorial operator is contained in some sector the same generic picture as for ordinary differential equations appears. Many systems, as reaction-diffusion systems, the Navier-Stokes equations, etc. fit into this abstract setup.

Example 1: Consider

$$
\partial_{t} u=\partial_{x}^{2} u+\alpha u-u^{3}
$$

for $x \in \mathbb{R}, t \geq 0, \alpha \in \mathbb{R}$, and $u(x, t) \in \mathbb{R}$ with $u(x, t)=u(x+2 \pi, t)$ and $u(x)=u(-x)$. The eigenfunctions of the operator $A$, defined by $A u=\partial_{x}^{2} u+\alpha u$, in

$$
X=\left\{u: \mathbb{R} \rightarrow \mathbb{R} \mid \int_{0}^{\pi} u^{2}(x) d x<\infty, u(x)=u(x+2 \pi), u(x)=u(-x)\right\}
$$

are given by $\varphi_{n}(x)=\cos n x, n \in \mathbb{N}_{0}$. The associated eigenvalues $\lambda_{n}=-n^{2}+\alpha$ are strictly negative for $\alpha<\alpha_{c}=0$. For $\alpha=\alpha_{c}$ one real eigenvalue, namely $\lambda_{0}$, crosses the imaginary axis. For $\alpha$ close to $\alpha_{c}$ all other eigenvalues are still strictly negative.

If symmetries are present, the picture looks different. From a mathematical point of view these situations are more interesting and there exists an extensive list of references (cf. [22, 23]). Moreover, it could be advantageous for the analysis of a system to construct artificial degeneracies by introducing new parameters such that more than one eigenvalue cross the imaginary axis for the same value of $\alpha$.

\section{Linear stability analysis on unbounded domains}

Now we come to the subject of this paper, dissipative systems, where the spatial variable lives in an unbounded cylindrical domain $\mathbb{R} \times \Sigma$, with $\Sigma \subset \mathbb{R}^{d}$ some bounded cross-section. Consider a translational invariant system

$$
\partial_{t} u=L_{\alpha}\left(\partial_{x}, \partial_{y}\right) u+N(u)
$$

for $(x, y) \in \mathbb{R} \times \Sigma$, with $\Sigma \subseteq \mathbb{R}^{d}$ a bounded cross-section with smooth boundary $\partial \Sigma$, and $u=u(x, y, t)$ not necessarily scalar-valued. The linear operator $L_{\alpha}$ depends on some parameter $\alpha \in \mathbb{R}$ and the term $N$ stands for the nonlinearity. For all $\alpha \in \mathbb{R}$ system (2) possesses the trivial solution $u \equiv 0$.

Example 2: Consider

$$
\partial_{t} u=\left(\partial_{x}^{2}+\partial_{y}^{2}\right) u+\alpha u-u^{3},\left.\quad u\right|_{y=0, \pi}=0
$$

with $t \geq 0,(x, y) \in \mathbb{R} \times(0, \pi)$, and $u(x, y, t) \in \mathbb{R}$. The operator $L_{\alpha}$ defined by $L_{\alpha}\left(\partial_{x}, \partial_{y}\right) u=\left(\partial_{x}^{2}+\partial_{y}^{2}\right) u+\alpha u$ possesses the eigenfunctions $\varphi_{n}(k)=\mathrm{e}^{i k x} \sin n y(k \in \mathbb{R}, n \in \mathbb{N})$. The associated eigenvalues $\lambda_{n}(k)=-k^{2}-n^{2}+\alpha$ are strictly negative for $\alpha<\alpha_{c}=1$, but in contrast to Example 1 for $\alpha>\alpha_{c}$ there are infinitely many eigenvalues with positive real part associated to a whole band $\left\{k \mid k^{2}<\alpha-\alpha_{c}\right\}$ of wavenumbers $k$.

For a general system (2) the situation is similar. Due to the translation invariance the eigenvalue problem

$$
L_{\alpha}\left(\partial_{x}, \partial_{y}\right) \mathrm{e}^{i k x} f_{n}(k, y)=\lambda_{n}(k) \mathrm{e}^{i k x} f_{n}(k, y),
$$

is satisfied by the Fourier modes $\mathrm{e}^{i k x} f_{n}(k, y)$, where $f_{n}(k, y)$ is some eigenfunction of $L_{\alpha}\left(i k, \partial_{y}\right)$ which lives on the bounded cross-section $\Sigma$ alone. For fixed Fourier wavenumber $k \in \mathbb{R}$ the spectrum is discrete, i.e. $n \in \mathbb{N}$, if $L_{\alpha}\left(i k, \partial_{y}\right)$ is 

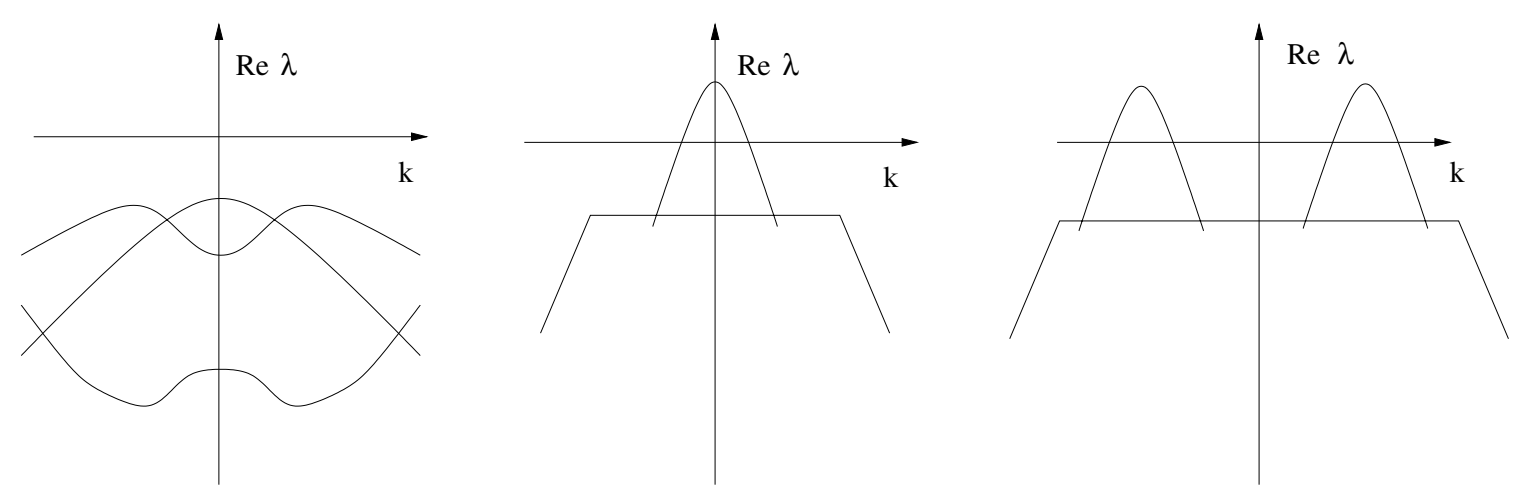

Fig. 4. a) Curve of eigenvalues for $\alpha<\alpha_{c}$, b) Instability at the wavenumber $k_{c}=0$, c) Instability at the wavenumber $k_{c} \neq 0$

some elliptic operator on the bounded cross-section $\Sigma$ which allows us to order the eigenvalues in such a way that $\operatorname{Re} \lambda_{n}(k) \geq \operatorname{Re} \lambda_{n+1}(k)$.

At a first sight there seems to be no possibility to classify the generic (and also the non generic) situations since with one eigenvalue crossing the imaginary axis infinitely many eigenvalues do the same. But such systems have some additional structure, namely that the eigenvalues $\lambda_{n}(k)$ can be drawn as functions over the wavenumbers $k$. If we assume that $u \equiv 0$ is stable for $\alpha<\alpha_{c}$ then for $\alpha<\alpha_{c}$ in the plane $(k, \operatorname{Re} \lambda)$ all curves $k \mapsto \operatorname{Re} \lambda_{n}(k)$ are below the $k$-axis, $\operatorname{Re} \lambda=0$. If instability occurs for $\alpha=\alpha_{c}$ generically one curve or a pair of complex conjugate curves $\lambda_{2}(k)=\overline{\lambda_{1}(-k)}$ touch the $k$-axis a) at a wavenumber $k=k_{c}=0$ or b) at a wavenumber $k=k_{c} \neq 0$ and since we consider a real-valued problem in the last case also at $k=-k_{c}$. Generically all other curves are strictly bounded away from the $k$-axis.

\section{Finite-dimensional bifurcation theory}

In order to get an idea how a bifurcation theory for unbounded domains has to look like we recall in this section basic facts from finite-dimensional bifurcation theory.

Consider the one-dimensional ordinary differential equation

$$
\partial_{t} u=\alpha u+\alpha_{2} u^{2}+\alpha_{3} u^{3}+\mathcal{O}\left(u^{4}\right),
$$

with $\alpha, \alpha_{j} \in \mathbb{R}$ and $u(t) \in \mathbb{R}$. For $\alpha<0$ the fixed point $u=0$ is stable. For $\alpha=0$ a real eigenvalue $\lambda_{1}(\alpha)=\alpha$ crosses the imaginary axis and a bifurcation of fixed points occurs. For $\alpha_{2} \neq 0$ we have a transcritical bifurcation drawn in Fig. 5a). Scaling $u(t)=\alpha \tilde{u}(|\alpha| t)=\alpha \tilde{u}(T)$ and neglecting higher order terms w.r.t. $\alpha$ gives the model

$$
\operatorname{sign}(\alpha) \partial_{T} \tilde{u}=\tilde{u}+\alpha_{2} \tilde{u}^{2}
$$

which could be analysed completely. The implicit function theorem allows us to prove the persistence of fixed points found by (4) in (3) under the higher order terms.

Very often due to symmetries the coefficient $\alpha_{2}$ vanishes. Then for $\alpha_{3} \neq 0$ we have a sub- or supercritical pitchfork bifurcation drawn in Figs. 5b) and c). Scaling $u(t)=|\alpha|^{1 / 2} \tilde{u}(|\alpha| t)=|\alpha|^{1 / 2} \tilde{u}(T)$ and neglecting higher order terms in $\alpha$ gives the model

$$
\partial_{T} u=u+\alpha_{3} u^{3}
$$

which again can be analysed completely.

The simplest model, where a pair of two complex conjugate eigenvalues crosses the imaginary axis is given in polar coordinates $\left(r \geq 0, \phi \in S^{1}\right)$ by

$$
\partial_{t} r=\alpha r+\alpha_{3} r^{3}+\mathcal{O}\left(r^{5}\right), \quad \partial_{t} \phi=1
$$
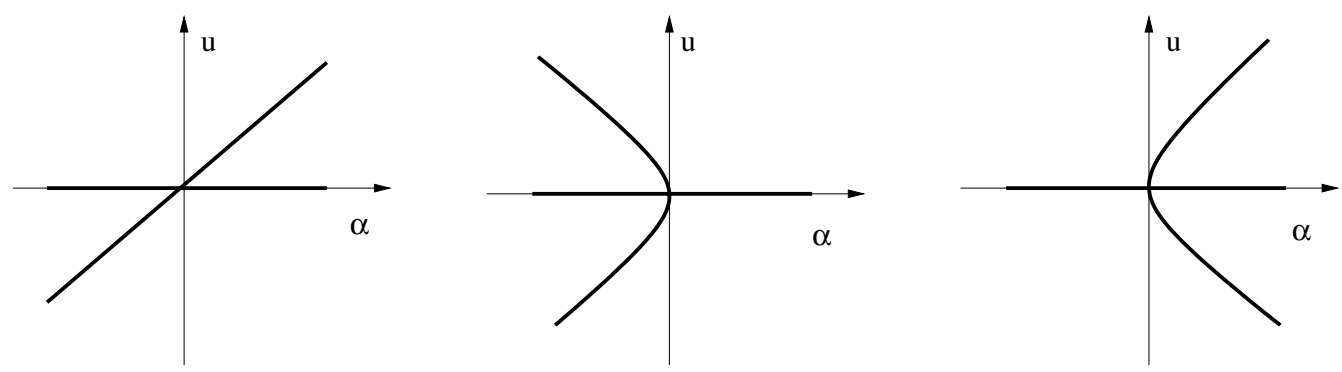

Fig. 5. a) Transcritical bifurcation, b) Subcritical pitchfork, c) Supercritical pitchfork 
For $\alpha=0$ a so called sub- or supercritical Hopf-bifurcation occurs [38], i.e. a bifurcation of a time-periodic solution. With the help of normal form transforms [24] it turned out that (6) is the standard form of all two-dimensional systems with this kind of instability.

Now assume that the more complicated system (1) with such kind of instabilities is considered. Then for instance in case of the generic instability a) explained in Section 2, i.e. in case of a real eigenvalue $\lambda_{1}$ crossing the imaginary axis, we write $u$ as a sum of the weakly unstable mode and the stable modes, i.e. $u=c_{1} \varphi_{1}+u_{r},\left(c_{1} \in \mathbb{R}\right)$, where $u_{r}$ lives in the closure of the span of the stable eigenfunctions $\left\{\varphi_{2}, \varphi_{3}, \ldots\right\}$. For the linear system $\dot{u}=A u$ all solutions are attracted by the one-dimensional set $E_{c}=\left\{u \mid u_{r}=0\right\}$, in which all solutions diverge to infinity.

For the nonlinear system and $\alpha$ close to $\alpha_{c}$ this attracting structure survives, no longer as a linear space, but as a manifold

$$
M_{c}=\left\{u=c_{1} \varphi_{1}+h\left(c_{1}\right) \mid h\left(c_{1}\right) \in \overline{\operatorname{span}\left\{\varphi_{2}, \varphi_{3}, \ldots\right\}}\right\}
$$

the so called center manifold which is tangential to $E_{c}$, i.e. $\left\|h\left(c_{1}\right)\right\| \leq C\left\|c_{1}\right\|^{2}$. The dynamics on $M_{c}$ is no longer trivial due to the nonlinear terms and the reduced system for $c_{1}$ is given in lowest order by the models (4) or (5). Due to this fact models (4) and (5) are called universal. Therefore, the full system (1) and the reduced system (3) show similar behavior.

In case of $u \in \mathbb{R}^{d}$ the center manifold theorem goes as follows (cf. [64]).

Theorem Consider (1). Assume that the function $f$ is in $C^{k}, k \geq 2$, in a neighborhood of $u=0$. Then there exists a $C^{k-1}$-manifold $M_{c}$, the center manifold, which is invariant under the flow and tangential to $E_{c}$. In case of no eigenvalues with positive real part the center manifold is attracting the solutions with some exponential rate.

This theorem also holds in infinite dimensions, i.e. for instance for dissipative partial differential equations, where the spatial variable lives in some bounded domain as in Example 1. See [65]. In [33] it was observed that it can also be used for elliptic PDE problems posed in spatially unbounded cylindrical domains. For this see also [28, 29, 41] and the proof of Theorem 7 .
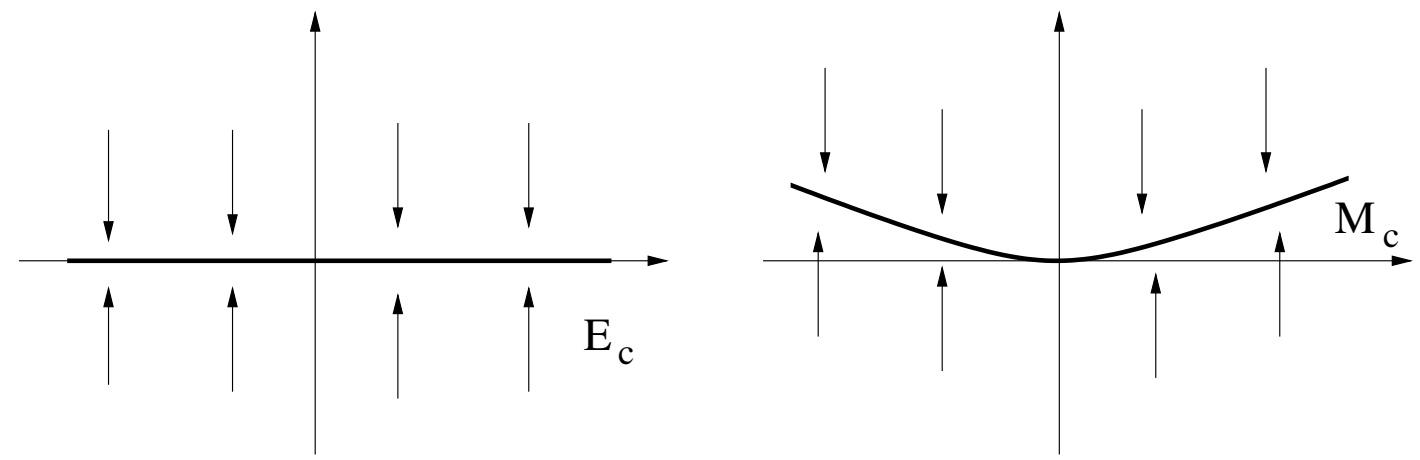

Fig. 6. The linear space $E_{c}$ and the center manifold $M_{c}$

\section{A zoo of universal modulation equations}

We come back to the subject of this paper, dissipative systems, where the spatial variable lives in an unbounded cylindrical domain $\mathbb{R} \times \Sigma$, where $\Sigma \subset \mathbb{R}^{d}$ is some bounded cross-section. Before we explain in more detail how we obtain the modulation equations as counterparts to the models (4)-(6) we give a survey of the modulation equations which occur generically. Depending on the knowledge of the reader it can be advantageous to read Section 6 before Section 5.

As explained in Section 3 there is generically one curve Re $\lambda_{1}$ or a pair of complex conjugate curves Re $\lambda_{1}$, Re $\lambda_{2}$ with $\lambda_{2}(k)=\overline{\lambda_{1}}(-k)$ which crosses the axis $\operatorname{Re} \lambda=0$ when an instability occurs. This can happen a) at a critical wavenumber $k_{c}=0$ or b) at a critical wavenumber $k_{c} \neq 0$.

The mathematical theory in the sense of Section 6 has been started ten years ago for the case b) $k_{c} \neq 0$. An overview about this case can be found in the original paper [59] or in the overview article [44]. By some perturbation ansatz which is explained for the Allen-Cahn equation as modulation equation in more detail in Section 6 one obtains the Ginzburg-Landau equation

$$
\partial_{T} A=c_{1} \partial_{X}^{2} A+c_{2} A+c_{3} A|A|^{2},
$$

with coefficients $c_{j} \in \mathbb{C}$ as the generic modulation equation. Mathematical theorems similar to ones in the next section has been proved first in $[11,15,34,47,48,51,66]$. The pseudo-orbit theorem has been carried out in [49]. With this 
method in [49] the global existence of all small solutions in the original systems has been established via the global existence of solutions for the Ginzburg-Landau equation. The proof of the uppersemicontinuity of attractors can be found in [42]. The whole theory has been carried out in greatest generality and applied to the Navier-Stokes equations [59].

Depending on the sign of $\operatorname{Re} c_{3}$ sub- $(>0)$ or supercritical $(<0)$ bifurcations occur.

In case of supercritical bifurcations complicated temporal behavior for the Ginzburg-Landau equation has been found for instance in [14]. Such bifurcations very often occur in pattern forming systems and the bifurcating solutions are usually not spatially localised. The construction of stationary, time-periodic, or modulating front solutions in the original system can be found in $[16,25,27,28]$ with the help of center manifold theory for elliptic PDEs and the Ginzburg-Landau equation. The stability question of these solutions is delicate. If they are not obviously unstable, the linearisation possesses continuous spectrum up to the imaginary axis. Since the nonlinear terms then usually do not possess an obvious sign a new method has to be used to establish the nonlinear stability. The so called diffusive stability method has been used to establish the stability of steady state solutions in $[6,12,30]$, of so called state mixing solutions in $[6,21]$, and of front solutions in $[7,17,20]$ for the Ginzburg-Landau equation. So far only the stability of the steady state solutions has been transfered to the original systems. In $[18,53]$ the stability of spatially periodic equilibria in the Swift-Hohenberg equation and in [58] the nonlinear stability of the Taylor vortices has been established.

In case of a subcritical bifurcation spatially localised solutions occur [2, 31]. The linearisation then possesses continuous spectrum strictly bounded away from the imaginary axis and so stability is determined by finitely many eigenvalues. In case of a degenerated subcritical bifurcation a so called generalised Ginzburg-Landau equation appears $[62]$

$$
\partial_{T} A=c_{1} \partial_{X}^{2} A+c_{2} A+c_{3} A|A|^{2}+c_{4}|A|^{2} \partial_{X} A+c_{5} A^{2} \partial_{X} \bar{A}+c_{6} A|A|^{4},
$$

with coefficients $c_{j} \in \mathbb{C}$. For this equation in [32] the exponential stability of a family of pulse-solutions has been shown. This exponentially stable structure can be recovered with the help of center manifold theory for elliptic PDEs and Floquet theory in form of an exponentially stable family of so called modulating pulse solutions [57].

Due to this extensive literature about bifurcations at the wavenumber $k_{c} \neq 0$ we will concentrate in following on bifurcations at the wavenumber $k_{c}=0$ which is more adequate for an introductory text. In case of a transcritical bifurcation of fixed points the so called KPP-equation $[19,36]$ occurs as the associated modulation equation

$$
\partial_{T} A=c_{1} \partial_{X}^{2} A+c_{2} A+c_{3} A^{2}, \quad A(X, T) \in \mathbb{R},
$$

where the $c_{j}$ are real-valued constants. The mathematical theory in the sense of Section 6 has been worked out in [56]. In case of a sub- or supercritical bifurcation of fixed points the so called Allen-Cahn equation occurs as the associated modulation equation

$$
\partial_{T} A=c_{1} \partial_{X}^{2} A+c_{2} A+c_{3} A^{3}, \quad A(X, T) \in \mathbb{R},
$$

where the $c_{j}$ are real-valued constants. So far for this equation the mathematical theory of modulation equations has not been carried out. Thus, we will use this case to explain the mathematical theory of modulation equations in Section 6 .

In case of a Hopf-bifurcation (in time) again the Ginzburg-Landau equation occurs as the associated modulation equation [37]. The mathematical theory in the sense of Section 6 has been worked out in [55]. The construction of time-periodic multi pulse solutions can be found in [3].

Another famous modulation equation, namely the Cahn-Hilliard equation

$$
\partial_{T} A=\partial_{x}^{2}\left(c_{1} \partial_{X}^{2} A+c_{2} A+c_{3} A^{3}\right), \quad A(X, T) \in \mathbb{R},
$$

where the $c_{j}$ are again real-valued constants, occurs if a marginal stable solution becomes sideband unstable at the critical wavenumber $k_{c}=0$ under some additional symmetry. The mathematical theory in the sense of Section 6 has been worked out in [60]. The construction of the stationary solutions can be found in [1].

Modulation equations can also be derived in domains $\mathbb{R}^{\tilde{d}} \times \Sigma$ with $\tilde{d} \neq 1$, i.e. in domains with more than one unbounded space direction. There exist approximation results [50, 52], but the whole theory of modulation equations in the sense of Section 6 can only be carried out if the attracting set of the original system shows the scaling properties which are necessary for the derivation of the modulation equation. See Theorem 2. This is as a general rule (not a mathematical statement) the case for a) systems with an instability at the wavenumber $k_{c}=0$ without restriction [55] on the space dimension $\tilde{d}$, b) for systems with an instability at the wavenumber $k_{c} \neq 0$ and $x \in \mathbb{R}$, and c) for anisotropic systems [59] with instability at the wavenumber $k_{c} \neq 0$ and no restriction on the space dimension $\tilde{d}$.

Then it is a successful tool which can be applied to describe the bifurcation scenario in such systems.

In situations, where the mathematical theory of modulation equations in the sense of Section 6 cannot be applied, it is not clear that the dynamics of the original system and the modulation equation have something to do with each other, and really there exists a counterexample of an original system with $x \in \mathbb{R}^{2}$ and $k_{c} \neq 0$ for which the dynamics behaves completely different than predicted by the formally correct derived modulation equation [52]. 
Again symmetry changes the picture of the generic modulation equations. Even in very simple situations, where the mathematical theory of modulation equations can be applied, it turned out that for instance for a Hopf-bifurcation at a wavenumber $k_{c} \neq 0$ in reflection symmetric systems bifurcation equations occur which still depend on the bifurcation parameter $\epsilon$. There are some constructions $[35,54]$ which try to eliminate the $\epsilon$-dependence, but in general this is not possible. However the resulting modulation equations can still be used to gain results about the original system like the global existence of all small solutions [59].

For a systematic discussion of the bifurcation scenario with euclidean symmetry in higher space dimensions see $[39,40]$.

\section{Mathematical theory of modulation equations}

We explain now the mathematical theory of modulation equations which allows us here to recover the dynamics of the Allen-Cahn equation in a more complicated system. We refrain from greatest generality and consider for simplicity a very special reaction-diffusion system. In contrast to the general case the assumptions below will allow us to do all calculations completely in $x$-space. But nevertheless, the analysis can be carried out in greatest generality as in [59]. For this see also the remarks at the end of this section. We mainly explain the results and in the appendix we give some of the proofs.

\subsection{The reaction-diffusion system}

In order to be precise in the following, we consider a system of reaction-diffusion equations on the real axis

$$
\partial_{t} u_{j}=\partial_{x}^{2} u_{j}+f_{j}\left(\alpha, u_{1}, \ldots, u_{d}\right) \quad(j=1, \ldots, d),
$$

with $t \geq 0, x \in \mathbb{R}, \alpha \in \mathbb{R}$, and $u_{j}(x, t) \in \mathbb{R}$. We assume for the nonlinear terms that $f_{j} \in C^{4}\left(\mathbb{R}^{d+1}, \mathbb{R}^{d}\right)$ and that $f_{j}(\alpha, 0, \ldots, 0)=0$ for all $\alpha \in \mathbb{R}$, i.e., that $u=\left(u_{1}, \ldots, u_{d}\right)=0$ is a stationary solution of $(7)$.

We are interested in the bifurcation scenario, when $u=0$ gets unstable. Therefore, we consider the linearisation which is given by

$$
\mathcal{A}=\partial_{x}^{2}+\left.D_{u} f\right|_{u=0}
$$

Due to the translation invariance of the system the eigenvectors of $\mathcal{A}$ are given by the Fourier modes $\varphi_{k, j} \mathrm{e}^{i k x}$ with $\varphi_{k, j} \in \mathbb{C}^{d}$ for $k \in \mathbb{R}$ and $j=1, \ldots, d$. The associated eigenvalues $\lambda(k, j)$ which we order such that $\operatorname{Re} \lambda(k, j) \geq \operatorname{Re} \lambda(k, j+1)$ satisfy

$$
\lambda(k, j)=\lambda(0, j)-k^{2} .
$$

Consequently the eigenfunctions $\varphi_{k, j}$ are independent of $k$ such that we can write $\varphi_{j}$ instead of $\varphi_{k, j}$.

We assume that $u=0$ is stable for $\alpha<0$. Due to (8) the fixed point $u=0$ gets unstable with respect to long wave perturbations, i.e., instability occurs at the critical wavenumber $k_{c}=0$. We are interested in a bifurcation of fixed points and so we assume that $\lambda(0,1)=0$ is a single eigenvalue for $\alpha=0$ and that all other eigenvalues are still strictly negative, i.e., there exists a $\beta>0$ such that $\operatorname{Re} \lambda(0, j)<-\beta$ for $j=2, \ldots, d$. In order to describe the small bifurcating solutions we introduce the small bifurcation parameter

$$
\epsilon^{2}=\lambda(0,1, \alpha)-\lambda(0,1,0) .
$$

As already mentioned the Allen-Cahn equation is the modulation equation for sub- or supercritical bifurcations at the critical wavenumber $k_{c}=0$ and so as for (5) some additional assumption on the nonlinear terms is needed. In order to formulate this assumption the eigenvectors of the adjoint matrix $A^{*}$ are denoted by $\varphi_{j}^{*}$. Then we assume

$$
\left\langle\varphi_{1}^{*},\left.D^{2} f\right|_{u=0}\left(\varphi_{1}, \varphi_{1}\right)\right\rangle_{\mathbb{C}^{d}}=0
$$

which guarantees that the Allen-Cahn equation is the modulation equation of the system. In order to separate the curve of biggest eigenvalues from the other ones projections $P_{1} u=\left\langle\varphi_{1}^{*}, u\right\rangle_{\mathbb{C}^{d}} \varphi_{1}$ and $P_{2}=I-P_{1}$ are defined. Without loss of generality (may be after some coordinate transform) it can be assumed that $\varphi_{1}=e_{1}=(1,0, \ldots, 0)$ and that $(7)$ can be written as

$$
\partial_{t} u_{1}=\epsilon^{2} u_{1}+\partial_{x}^{2} u_{1}+N_{1}(u), \quad \partial_{t} v=Q v+\partial_{x}^{2} v+N_{2}(u)
$$

where $N_{j}(u)=P_{j}\left(f(u)-\left.D_{u} f\right|_{u=0} u\right), Q \in \mathbb{R}^{(d-1) \times(d-1)}$ with eigenvalues $\lambda(0, j)$ for $j=2, \ldots, d$. For notational simplicity $v(x, t) \in \mathbb{R}^{d-1}$ is identified with $(0, v(x, t)) \in \mathbb{R}^{d}$.

\subsection{The attracting set}

As already explained modulation equations are the infinite-dimensional counterparts to the finite-dimensional amplitude equations on the center manifolds. The center manifold theorem justifies the models (4) - (6) since it proves that 
(4) - (6) describes the dynamics of the original system in an attracting set. In the same spirit the mathematical theory of modulation equations shows that the modulation equations describe approximately the dynamics of the original system in an attracting set. In order to see the structure of this attracting set we look at the linearised problem first. Obviously for $j=2, \ldots, d$

$$
\sup _{x \in \mathbb{R}}\left|u_{j}(x, t)\right| \leq C \mathrm{e}^{\lambda(0,2) t} \rightarrow 0
$$

for $t \rightarrow \infty$ holds. Hence, except of the $u_{1}$-component all other components are damped with some exponential rates. In general except of some small neighborhood of the critical wavenumber $k_{c}=0$ it will not be possible to separate the $u_{1}$ component from the other components for all wavenumbers $k$. But this is no problem since outside this neighborhood the Fourier modes of $u_{1}$ are exponentially damped. The difficulty comes from the fact that there is no spectral gap for the modes belonging to the $u_{1}$-variable, and so it is not clear which modes are of interest for the nonlinear problem. It turned out that the component $u_{1}$ develops in such a way that there is a certain mode-distribution after a certain time and that this mode-distribution can be used for the description of the attracting set also in the nonlinear problem. The solutions $u_{1}$ of

$$
\partial_{t} u_{1}=\epsilon^{2} u_{1}+\partial_{x}^{2} u_{1}
$$

stay $\mathcal{O}(1)$-bounded for $t \in\left[0, T_{0} / \epsilon^{2}\right]$ for each fixed $T_{0}>0$. Since in Fourier space

$$
\epsilon^{-1} \hat{u}_{1}\left(k, T_{0} / \epsilon^{2}\right)=\epsilon^{-1} \mathrm{e}^{\epsilon^{2} T_{0} / \epsilon^{2}} \mathrm{e}^{-k^{2} T_{0} / \epsilon^{2}} \hat{u}_{1}(k, 0)=\epsilon^{-1} \mathrm{e}^{T_{0}} \mathrm{e}^{-(k / \epsilon)^{2} T_{0}} \hat{u}_{1}(k, 0)
$$

at a time $t=T_{0} / \epsilon^{2}$ the Fourier modes are concentrated in an $\mathcal{O}(\epsilon)$-neighborhood of the wavenumber $k_{c}=0$.

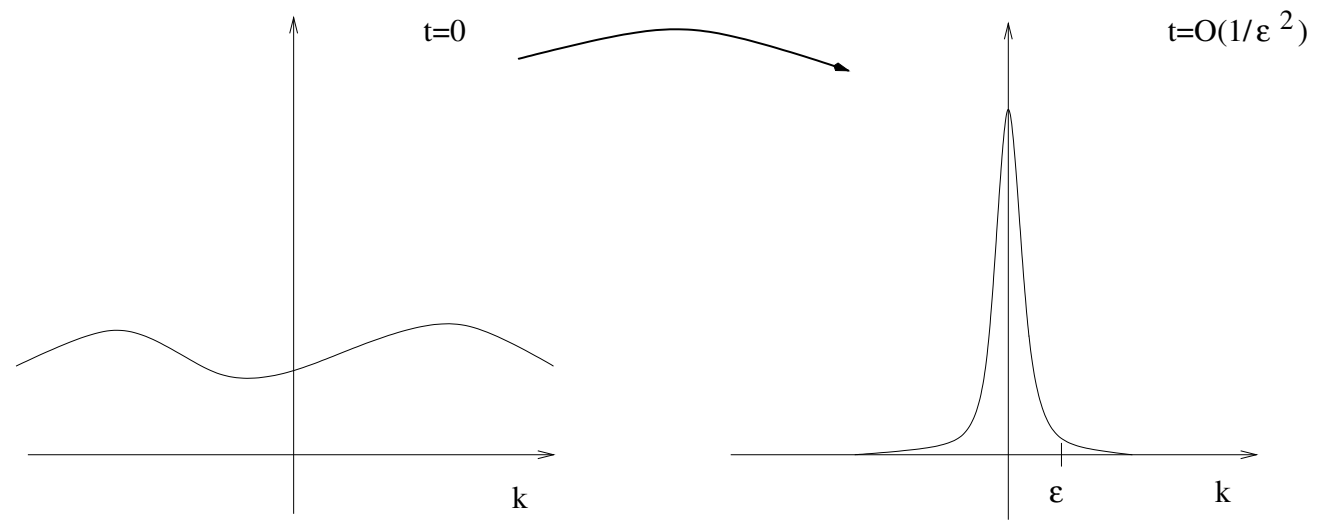

Fig. 7. b) Concentration of the Fourier modes at $k_{c}=0$ at a time $t=T_{0} / \epsilon^{2}$ to a) arbitrary initial conditions in Fourier space

As a consequence in physical space the function

$$
u_{1}\left(x, T_{0} / \epsilon^{2}\right)=\epsilon^{-1} \int_{\mathbb{R}} \mathrm{e}^{T_{0}} \mathrm{e}^{-(k / \epsilon)^{2} T_{0}} \hat{u}_{1}(k, 0) \mathrm{e}^{i k x} d k=\int_{\mathbb{R}} \mathrm{e}^{T_{0}} \mathrm{e}^{-K^{2} T_{0}} \hat{u}_{1}(\epsilon K, 0) \mathrm{e}^{i K X} d K
$$

varies as a function of $X=\epsilon x$ slowly in space. An $\mathcal{O}(1)$-boundedness of the derivatives $\partial_{X}^{n} u_{1}=\epsilon^{-n} \partial_{x}^{n} u_{1}$ is the counterpart to the concentration in Fourier space. From the explicit formula

$$
u_{1}\left(x, T_{0} / \epsilon^{2}\right)=\frac{\epsilon}{\sqrt{2 \pi T_{0}}} \int_{\mathbb{R}} \mathrm{e}^{(\epsilon(x-y))^{2} /\left(4 T_{0}\right)} u_{0}(y) d y
$$

for all $n \in \mathbb{N}$ and all $T_{0}>0$ there exist $\epsilon_{0}>0$ and $C>0$ such that for all $\epsilon \in\left(0, \epsilon_{0}\right)$ we have

$$
\sup _{X \in \mathbb{R}}\left|\partial_{X}^{n} u_{1}\left(X / \epsilon, T_{0} / \epsilon^{2}\right)\right| \leq C T_{0}^{-n / 2} \sup _{x \in \mathbb{R}}\left|u_{1}(x, 0)\right| \text {. }
$$

Hence, at a time $t=T_{0} / \epsilon^{2}$ the solutions $u$ of the linearisation of (7) can be written as

$$
u\left(x, T_{0} / \epsilon^{2}\right)=A(\epsilon x) e_{1}+R(x)
$$

with $P_{1} R=0, A(\epsilon x) \in \mathbb{R}$,

$$
\|A\|_{C_{b}^{n}}=\sup _{j=0, \ldots, n} \sup _{X \in \mathbb{R}}\left|\partial_{X}^{j} A(X)\right|=\mathcal{O}(1) \quad \text { and } \quad\|R\|_{C_{b}^{n}}=\mathcal{O}\left(\mathrm{e}^{-C / \epsilon}\right)
$$




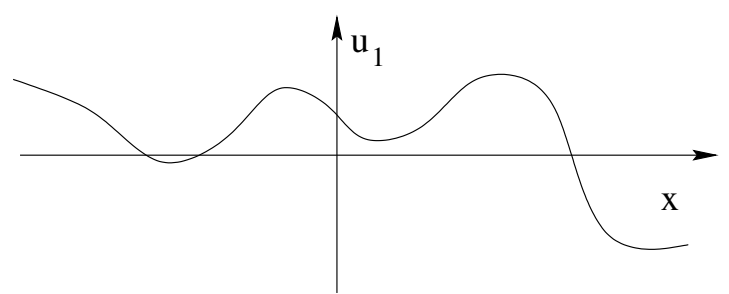

Fig. 8. b) Long wave solutions at $t=T_{0} / \epsilon^{2}$ to a) arbitrary initial conditions

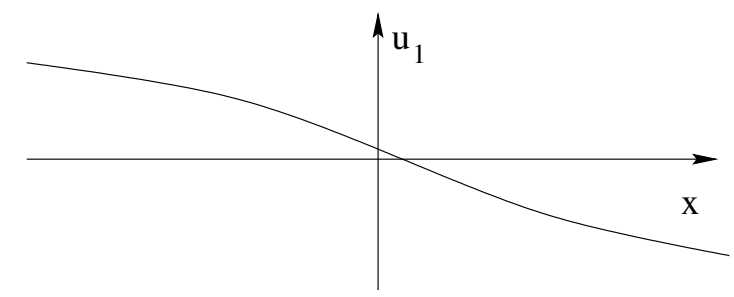

$1 / \varepsilon$

for all $n \in \mathbb{N}$ as $\epsilon \rightarrow 0$. It turned out that this is also the right characterisation for the nonlinear system (7), except of some small modification. As in the center manifold theorem the variables $u_{2}, \ldots, u_{d}$ are no longer exponentially damped, but of order $\mathcal{O}\left(\left\|u_{1}\right\|^{2}\right)$. From (5) it is already known that the solutions bifurcating from $u=0$ are of order $\mathcal{O}(\epsilon)$.

Theorem 2: Fix $C_{1}>0$ and $m \in \mathbb{N}$. Then there exist $T_{0}, C_{2}, \epsilon_{0}>0$, such that for all $\epsilon \in\left(0, \epsilon_{0}\right)$ the following holds: Let $u_{0} \in C_{b}^{0}$ with $\left\|u_{0}\right\|_{C_{b}^{0}}<C_{1} \epsilon$. Then the solution $u$ of $(7)$ with $\left.u\right|_{t=0}=u_{0}$ can be written as

$$
\left.u(x, t, \epsilon)\right|_{t=T_{0} / \epsilon^{2}}=\epsilon A(\epsilon x) e_{1}+\epsilon^{2} R(x, \epsilon),
$$

where $\|A\|_{C_{b}^{m}} \leq C_{2}$ and $\|R\|_{C_{b}^{0}} \leq C_{2}$.

Proof: See subsection 7.2 .

By looking for instance at the family of stationary solutions $\tilde{I}_{1}$ constructed in subsection 6.5 it follows that stronger concentration of Fourier modes is not true for the nonlinear system (7), i.e., Theorem 12 is optimal in this sense. Here and in the following the space $C_{b}^{0}$, wherein the original system is handled, could be replaced by some $C_{b}^{n}$.

\subsection{Approximation by the Allen-Cahn equation}

Theorem 2 guarantees the existence of an attracting set similar to the center manifold. The idea is to find a universal model similar to the models (4)-(6) which describe the dynamics of the solutions bifurcating in systems with this kind of instability.

Due to the representations of the solutions in Theorem 2 we make the ansatz

$$
u_{1}=\epsilon A\left(\epsilon x, \epsilon^{2} t\right) e_{1}+\epsilon^{2} B\left(\epsilon x, \epsilon^{2} t\right)+\mathcal{O}\left(\epsilon^{3}\right),
$$

where $B$ with $P_{1} B=0$ is also a scaled function. Inserting this into (7) shows

$$
\begin{aligned}
& \partial_{T} A=\partial_{X}^{2} A+A+\left.\epsilon^{-1} P_{1} D^{2} f\right|_{u=0}\left(A e_{1}, A e_{1}\right)+\left.2 P_{1} D^{2} f\right|_{u=0}\left(A e_{1}, B\right)+\left.P_{1} D^{3} f\right|_{u=0}\left(A e_{1}, A e_{1}, A e_{1}\right)+\mathcal{O}(\epsilon), \\
& \mathcal{O}\left(\epsilon^{2}\right)=Q B+\left.P_{2} D^{2} f\right|_{u=0}\left(A e_{1}, A e_{1}\right)+\mathcal{O}(\epsilon) .
\end{aligned}
$$

Due to the assumption (9) we have that $\left.\epsilon^{-1} P_{1} D^{2} f\right|_{u=0}\left(A e_{1}, A e_{1}\right)=0$, and so we define the approximation

$$
\psi_{\epsilon}(A)=\epsilon A\left(\epsilon x, \epsilon^{2} t\right) e_{1}+\epsilon^{2} B\left(\epsilon x, \epsilon^{2} t\right),
$$

where $A$ satisfies the Allen-Cahn equation

$$
\partial_{T} A=\partial_{X}^{2} A+A+c_{3} A^{3}
$$

with

$$
c_{3}=\left\langle e_{1},\left.D^{3} f\right|_{u=0}\left(e_{1}, e_{1}, e_{1}\right)\right\rangle_{\mathbb{R}^{d}}-2\left\langle e_{1},\left.D^{2} f\right|_{u=0}\left(e_{1},\left.Q^{-1} P_{2} D^{2} f\right|_{u=0}\left(e_{1}, e_{1}\right)\right)\right\rangle_{\mathbb{R}^{d}}
$$

and where $B$ satisfies

$$
B=-\left.Q^{-1} P_{2} D^{2} f\right|_{u=0}\left(e_{1}, e_{1}\right) A^{2} .
$$

The stationary solutions of the Allen-Cahn equation (2) can be analysed completely in the phase plane $\left(A, \partial_{X} A\right)$. As for (4) or (5) the implicit function theorem has to be used to prove their persistence in the original system (7). A successful method to do so is center manifold theory for elliptic PDEs. For an example see subsection 6.5. But the models (4)-(6) also describe the dynamics of (3) approximately. This follows immediately from Gronwall's inequality. So in order to prove that the Allen-Cahn equation (16) is a good model an approximation result has to be shown.

Theorem 3: For all $R_{1}, T_{1}, d>0$ there exist $C_{1}, \epsilon_{0}>0$ such that for all $\epsilon \in\left(0, \epsilon_{0}\right)$ the following holds: Let $A$ be a solution of (12) with $\sup _{T \in\left[0, T_{1}\right]}\|A(T)\|_{C_{b}^{3}} \leq R_{1},\left.A\right|_{T=0}=A_{0}$, and $u_{0} \in C_{b}^{0}$ an initial condition of (7) with

$$
\left\|u_{0}-\psi_{\epsilon}\left(A_{0}\right)\right\|_{C_{b}^{0}} \leq d \epsilon^{2}
$$


then there exists a unique solution $u$ of $(7)$ with $\left.u\right|_{t=0}=u_{0}$ and

$$
\sup _{t \in\left[0, T_{1} / \epsilon^{2}\right]}\left\|u(t)-\psi_{\epsilon}\left(A\left(\epsilon^{2} t\right)\right)\right\|_{C_{b}^{0}} \leq d \epsilon^{2}
$$

Proof: See subsection 7.3 .

\subsection{The pseudo-orbit approximation}

The attractivity and the approximation theorem can be improved and condense in the so called pseudo-orbit approximation theorem. With this theorem solutions of the original system can be approximated for all times by the solutions of the Allen-Cahn equation.

An approximation by a single solution of the Allen-Cahn equation is only possible for a finite time interval, but an approximation by a so called pseudo-orbit is possible for all $t \in(0, \infty)$.

Definition 1: Let $T_{1}>0$ and $\kappa>0$. We call a function $A=A(T)$ a $\left(T_{1}, \kappa\right)$-pseudo-orbit in the Banach space $Y$ for (2) if for all $n \in \mathbb{N}$ the relations

$$
\begin{aligned}
& A\left((n-1) T_{1}+\tau\right)=\mathcal{G}_{\tau}(A((n-1) T)) \quad \text { for all } \tau \in\left[0, T_{1}\right), \text { and } \\
& \left\|A\left(n T_{1}+0\right)-\mathcal{G}_{T_{1}}\left(A\left((n-1) T_{1}\right)\right)\right\|_{Y} \leq \kappa
\end{aligned}
$$

hold, where $\mathcal{G}_{T}$ is the semigroup associated with $(2)$ and $A(T+0)=\lim _{\tau \rightarrow T, \tau>T} A(\tau)$.

Thus, a pseudo-orbit solves the Allen-Cahn equation on finite time-intervals of length $T_{1}$ and at the end of each time-interval it makes some jump of size less than $\kappa$. The idea is: at the end of each approximation interval some error $u\left(T_{1} / \epsilon^{2}\right)-\psi_{\epsilon}\left(A_{0}\left(T_{1}\right)\right)$ has accumulated. Due to the attractivity theorem (Theorem 2) there exists an initial condition $A_{1}$ of the Allen-Cahn equation for which the error $u\left(T_{1} / \epsilon^{2}\right)-\psi_{\epsilon}\left(A_{1}\right)$ is smaller than the error $u\left(T_{1} / \epsilon^{2}\right)-\psi_{\epsilon}\left(A_{0}\left(T_{1}\right)\right)$. The possible difference $A_{0}\left(T_{1}\right)-A_{1}$ defines the size $\kappa$ of the jump. It turned out that this jump $\kappa$ is small.

Theorem 4: For all $C_{0}, C_{1}, T_{1}>0$ there exist positive constants $\epsilon_{0}, C_{2}$, and $T_{0}$ such that for all $\epsilon \in\left(0, \epsilon_{0}\right]$ the following is true:

For all initial conditions $u_{0}$ with $\left\|u_{0}\right\|_{C_{b}^{0}} \leq C_{0} \epsilon$ there is a $\left(T_{1}, C_{2} \epsilon^{1 / 4}\right)$-pseudo-orbit $A$ for (12) in $C_{b}^{1}$ which approximates $u(t)=\mathcal{S}_{t}^{\epsilon}\left(u_{0}\right)$ as

$$
\left\|u(t)-\psi_{\epsilon}\left(A\left(\epsilon^{2} t-T_{0}\right)\right)\right\|_{C_{b}^{0}} \leq C_{2} \epsilon^{5 / 4} \quad \text { for all } t \geq T_{0} / \epsilon^{2}
$$

as long as $\left\|A\left(\epsilon^{2} t\right)\right\|_{C_{b}^{1}} \leq C_{1}$, where $\mathcal{S}_{t}^{\epsilon}$ is the semigroup associated with (7).

Proof: This theorem follows by some technical improvements of the Theorems 2 and 3 as in [49], where the Ginzburg-Landau equation is handled as modulation equation in case of an instability at the wavenumber $k_{c} \neq 0$.

As a consequence of this theorem there is a correspondence between the pseudo-orbits in the modulation equation, here the Allen-Cahn equation, and the solutions in the attracting set of the original system (7). A generalised version $[42,59]$ of this theorem can be used to prove that an $\mathcal{O}(1)$-neighborhood of the unstable origin of $(7)$ is mapped into an $\mathcal{O}(\epsilon)$-neighborhood of the origin. In case of an exponentially stable structure in the modulation equation we can follow the solutions in the original system for all times without proving the existence and exponential stability of such structure in the original system. This theorem is used in the next section to analyse the bifurcation scenario of (7), but first it is applied to prove the global existence in time of all small solutions in the original system (7) with the help of the existence of an exponentially absorbing ball for the Allen-Cahn equation (2).

Assume for the rest of the paper that $c_{3}<0$ or without loss of generality that $c_{3}=-1$. Then by the maximum principle $B_{l}(0)<A(X, 0)<B_{u}(0)$ for all $X \in \mathbb{R}$ yields $B_{l}(T)<A(X, T)<B_{u}(T)$ for all $X \in \mathbb{R}$ and all $T \geq 0$, where the $B_{j}(T) \in \mathbb{R}$ satisfy the ordinary differential equation

$$
\partial_{T} B_{j}=B_{j}-B_{j}^{3} .
$$

As a consequence it follows $\limsup _{T \rightarrow \infty}\left|B_{j}(T)\right| \leq 1$ and $\limsup _{T \rightarrow \infty} \sup _{X \in \mathbb{R}}|A(X, T)| \leq 1$. Due to the smoothing properties of the linear semigroup the existence of a $C>0$ follows such that

$$
\limsup _{T \rightarrow \infty}\|A(\cdot, T)\|_{C_{b}^{1}} \leq C .
$$

Since the Allen-Cahn equation possesses an exponentially aborbing ball it follows

$$
\limsup _{T \rightarrow \infty}\|A(\cdot, T)\|_{C_{b}^{1}} \leq 2 C
$$

for the pseudo-orbits $A$ if $\epsilon_{0}>0$ is sufficiently small. Then Theorem 4 yields

$$
\limsup _{t \rightarrow \infty} \sup _{x \in \mathbb{R}}|u(x, t)| \leq C \epsilon
$$


for another constant $C$ independent of $\epsilon$ and for all solutions $u$ of $(7)$ with initial conditions $\left.u\right|_{t=0}=u_{0}$ in an $\mathcal{O}(\epsilon)$ neighborhood of the origin.

\subsection{Conclusions: Dynamics of interface solutions}

In this section we sketch some ideas how the above theory can be applied. The Allen-Cahn equation

$$
\partial_{T}=\partial_{X}^{2} A+A-A^{3}
$$

possesses two exponentially stable steady state solutions $A= \pm 1$. With the help of the pseudo-orbit theorem the dynamics of (7) can be controlled by the pseudo-orbits of the Allen-Cahn equation alone. Due to the exponential stability of $A= \pm 1$ the pseudo-orbits stay close to $A= \pm 1$ for all times. By Theorem 4 the associated solutions $u$ of (7) stay close to $\psi_{\epsilon}( \pm 1)$ for all times. With the implicit function theorem this result can be improved and close to $\psi_{\epsilon}( \pm 1)$ the existence of two exponentially stable spatially homogenous steady states $u_{ \pm}$for (7) can be shown.

The next nontrivial solutions of (2) are given by the two one-dimensional families of equilibria

$$
I_{1}=\left\{A_{c}=\tanh ( \pm(x+c) / \sqrt{2}) \mid c \in \mathbb{R}\right\}
$$

connecting the exponential stable states $A= \pm 1$ for $x \rightarrow \pm \infty$. The linearisation around $A_{c}$ for fixed $c$ possesses one zero eigenvalue corresponding to the eigenfunction $\partial_{x} A_{c}$. The rest of the spectrum is contained in some sector in the left half plane strictly bounded away from the imaginary axis. See [26]. The one-dimensional center manifold (see Theorem 1) to the zero eigenvalue is obviously given by $I_{1}$. Thus, we have:

Lemma 5: For all $C_{1}>0$ there exists a $C_{2}>0$ such that from

$$
\inf _{c \in \mathbb{R}}\left\|A(\cdot, 0)-A_{c}(\cdot)\right\|_{C_{b}^{1}}<C_{2}
$$

it follows for the solution $A$ of (2) that

$$
\inf _{c \in \mathbb{R}}\left\|A(\cdot, T)-A_{c}(\cdot)\right\|_{C_{b}^{1}} \leq C_{1} \mathrm{e}^{-\gamma T},
$$

for a constant $\gamma>0$ independent of $C_{1}$.

Applying the pseudo-orbit theorem (Theorem 4) shows

Lemma 6: There exists a $C_{1}>0$ such that for all $C_{2}>0$ we have an $\epsilon_{0}>0$ such that for all $\epsilon \in\left(0, \epsilon_{0}\right)$ the following holds. From

$$
\inf _{c \in \mathbb{R}}\left\|u(\cdot, 0)-\psi_{\epsilon}\left(A_{c}(\epsilon \cdot)\right)\right\|_{C_{b}^{0}}<C_{1} \epsilon
$$

it follows for the solution $u$ of (16) that

$$
\limsup _{t \rightarrow \infty} \inf _{c \in \mathbb{R}}\left\|u(\cdot, t)-\psi_{\epsilon}\left(A_{c}(\epsilon \cdot)\right)\right\|_{C_{b}^{0}} \leq C_{2} \epsilon .
$$

Proof: A solution $u$ of the original system is approximated by a pseudo-orbit in the Allen-Cahn equation. The constant $C_{1}$ comes from the fact that $u$ has to start close to $\psi_{\epsilon}\left(I_{1}\right)$. By continuity the initial conditions of the pseudoorbit can be chosen in the attracting neighborhood of $I_{1}$. By Lemma 6.5 for each $C_{2}>0$ there exists a $T_{1}>0$ such that the solution $A_{1}\left(T_{1}\right)$ is $C_{2} / 2$-close to $I_{1}$. By choosing $\epsilon$ sufficiently small the pseudo-orbit solution stay $C_{2}$-close to $I_{1}$. Going back with Theorem 6.4 into the original system (7) proves the result.

For these stationary solutions the result can be improved by using the reflection symmetry of the system, spatial dynamics and the center manifold theorem for elliptic PDEs.

Theorem 7: There exist $C_{1}, C_{2}, \gamma, \epsilon_{0}>0$ such that for all $\epsilon \in\left(0, \epsilon_{0}\right)$ the following holds. There exists a onedimensional family of stationary solutions

$$
\tilde{I}_{1}=\left\{u(x, t)=U\left(x-x_{0}\right) \mid x_{0} \in \mathbb{R}, \lim _{\xi \rightarrow \pm \infty} U(\xi)=u_{ \pm}\right\}
$$

This family is exponentially stable, i.e., from

$$
\inf _{x_{0} \in \mathbb{R}}\left\|u(\cdot, 0)-U\left(\cdot+x_{0}\right)\right\|_{C_{b}^{0}} \leq C_{1} \epsilon
$$

it follows

$$
\inf _{x_{0} \in \mathbb{R}}\left\|u(\cdot, t)-U\left(\cdot+x_{0}\right)\right\|_{C_{b}^{0}} \leq C_{2} \epsilon \mathrm{e}^{-\gamma t} .
$$

Proof: See subsection 7.4.

The strength of the pseudo-orbit theorem can be seen by considering solutions which cannot be transfered to the original system with the help of the center manifold theorem for elliptic PDEs, i.e. by considering solutions which are not of permanent form in the modulation equation. 


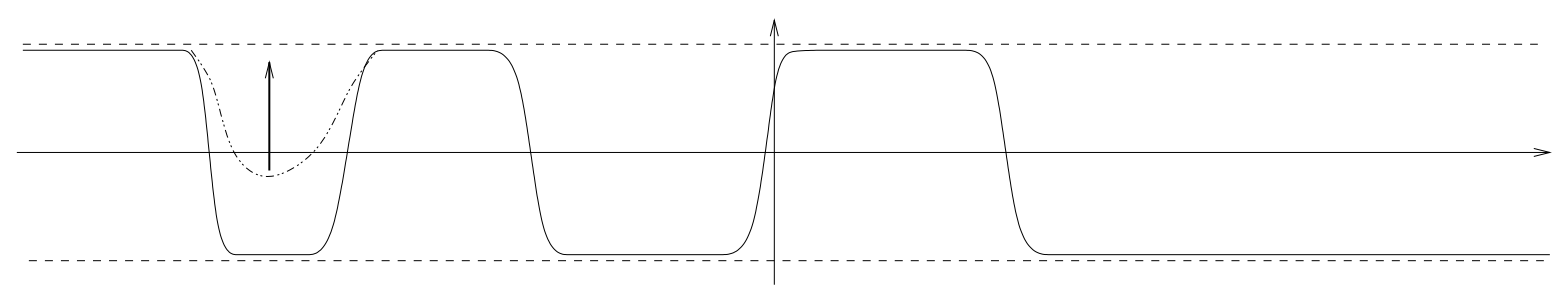

Fig. 9. An interface solution with 5 interfaces. Annihilation of two interfaces to an interface solution with 3 interfaces

For the Allen-Cahn equation solutions with more than one interface are examples of such solutions. These solutions consists of almost flat regions, where they are close to the exponentially stable states \pm 1 . In between there are the interface regions, where the solutions connect the stable state -1 with the stable state 1 or vice versa. A detailed description of the dynamics of these solutions and an exact definition can be found in [46].

We define classes of functions with $m$ interfaces:

$$
I_{m}=\left\{A \in C_{b}^{0}(\mathbb{R}, \mathbb{R}) \mid A \text { interface function with } m \text { zeros, } \lim _{X \rightarrow \pm \infty} A(X)=1 \text { or }-1\right\} .
$$

Then from the analysis in [46] and the literature cited there, it is known that the interfaces annihilate. Moreover, it is known that this happens in very robust way, i.e., small perturbations do not change the dynamics. With the help of the maximum principle the following global result can be obtained:

$$
\lim _{t \rightarrow \infty} A(\cdot, t)= \pm 1, \quad \text { if } \quad A(\cdot, 0) \in I_{2 m}, \quad \lim _{t \rightarrow \infty} A(\cdot, t) \in I_{1}, \quad \text { if } \quad A(\cdot, 0) \in I_{2 m+1}
$$

for $m \in \mathbb{N}$. Due to these facts with the help of the pseudo-orbit theorem (Theorem 4) and Theorem 7 it follows

Theorem 8: Fix $m \in \mathbb{N}$. Then there exist $C_{1}, C_{2}, \gamma, \epsilon_{0}>0$ such that for all $\epsilon \in\left(0, \epsilon_{0}\right)$ the following holds. From

$$
\inf _{A \in I_{m}}\left\|u(\cdot, 0)-\psi_{\epsilon}(A(\epsilon \cdot))\right\|_{C_{b}^{0}} \leq C_{1} \epsilon
$$

it follows for $m \in 2 \mathbb{N}$ that

$$
\left\|u(\cdot, t)-u_{ \pm}\right\|_{C_{b}^{0}} \leq C_{2} \epsilon \mathrm{e}^{-\gamma t}
$$

and for $m \in 2 \mathbb{N}+1$ that

$$
\inf _{U \in \tilde{I}_{1}}\|u(\cdot, t)-U(\cdot)\|_{C_{b}^{0}} \leq C_{2} \epsilon \mathrm{e}^{-\gamma t} .
$$

Thus, it is possible to prove such a result in a system for which the maximum principle used for (12) does not hold. A single application of the approximation result (Theorem 3) instead of the pseudo-orbit approximation would also allow us to prove such a result, but then the size of $\epsilon_{0}>0$ would be extremely small since the time for the annihilation of the interfaces goes exponentially $\left(\mathcal{O}\left(\mathrm{e}^{C L}\right)\right)$ with the distance $L$ of the interfaces.

The pseudo-orbit theorem also allows us to transfer another result which can be found in [46]. For the AllenCahn equation it has been shown that by separating the interfaces in the right way, i.e., making the distances bigger and bigger for $x \rightarrow \pm \infty$ it is possible to have an annihilation of interfaces for all times, i.e. infinitely many annihilations. Again this result is robust under small perturbations. With the help of the pseudo-orbit theorem (Theorem 4) it is obvious that also for the original system (7) we have annihilations of interfaces for all times, i.e. infinitely many annihilations.

\subsection{Generalizations}

First of all there is no need to restrict the analysis to one unbounded space direction, i.e., also $x \in \mathbb{R}^{\tilde{d}}$ with $\tilde{d} \neq 1$ can be handled.

Moreover, the restriction to reaction-diffusion systems can be dropped. A possible set $(\tilde{d}=1)$ of assumptions would the ones from [59], where assumption (B2) has to be replaced by

(B2) There exist $\rho_{0}, \epsilon_{0}>0$, such that for $0<\epsilon<\epsilon_{0}$ and $|k|<\rho_{0}$ we have a curve $\lambda\left(\cdot, \epsilon^{2}\right) \in C^{3}\left(\left(-\rho_{0}, \rho_{0}\right)\right.$, $\left.\mathbb{C}\right)$ of single eigenvalues and a curve of eigenfunctions $k \mapsto \varphi_{1}\left(k, \varepsilon^{2}\right)$ of $\mathcal{A}$ such that $k \mapsto \varphi_{1}\left(k, \varepsilon^{2}\right) \in C^{3}\left(\left(-\rho_{0}, \rho_{0}\right), W_{2}\right)$. Around $k_{c}=0$ the curve of eigenvalues has the expansion

$$
\lambda\left(k, \varepsilon^{2}\right)=i v_{1} k+\varepsilon^{2} \lambda_{0}+k^{2} \lambda_{2}+\mathcal{O}\left(\varepsilon^{3}+|k|^{3}\right)
$$

with $\lambda_{0}>0$ and $\lambda_{2}<0$. Moreover, there exists an $\epsilon$-independent constant $\sigma_{0}>0$ such that for $0<\epsilon<\epsilon_{0}$ the spectrum $\Sigma$ of $\mathcal{A}$ in $Z$ satisfies $\sup \Re\left(\Sigma^{-}\right)<-2 \sigma_{0}$, where $\Sigma^{-}$consists of the spectrum of $\mathcal{A}$ without the curves in $\Sigma^{+}=\left\{\bigcup_{k: k \mid<\rho_{0}} \lambda\left(k, \epsilon^{2}\right)\right\}$ 
Here $W_{2}$ and $Z$ are specified Banach spaces in [59]. In general $\varphi_{1}(k)$ depends on $k$ such that (9) has to be replaced by

$$
\left\langle\varphi_{1}^{*}(0),\left.D^{2} \mathcal{N}\right|_{u=0}\left(\varphi_{1}(0), \varphi_{1}(0)\right)\right\rangle_{W_{0}}=0,
$$

where $\mathcal{N}$ and $W_{0}$ are also specified in [59]. Then a generalized Allen-Cahn equation

$$
\partial_{T} A=\lambda_{0} A-\lambda_{2} \partial_{X}^{2} A+c_{3} A^{3}+c_{4} \partial_{X}\left(A^{2}\right)
$$

is obtained. The term $c_{4} \partial_{X}\left(A^{2}\right)$ vanishes for instance under the additional assumption of reflection symmetry.

\section{Appendix}

\subsection{The variation of constant formula and Gronwall's inequality}

Consider the differential equation

$$
\partial_{t} u=\mathcal{A} u+f(u) .
$$

Then the variation of constant formula $[9,26]$ is given by

$$
u(t)=\mathrm{e}^{\mathcal{A} t} u(0)+\int_{0}^{t} \mathrm{e}^{\mathcal{A}(t-s)} f(u(s)) d s .
$$

Suppose that the inequality

$$
u(t) \leq C_{1}+C_{2} \int_{0}^{t} u(s) d s
$$

holds. Then Gronwall's inequality $[9,26]$ shows

$$
u(t) \leq C_{1} \mathrm{e}^{C_{2} t} .
$$

\subsection{Proof of Theorem 2}

The situation is complicated through the fact that we have quadratic terms and so the long time existence $\mathcal{O}\left(1 / \epsilon^{2}\right)$ needs some additional effort. This situation is already handled in several papers $[5,15,51,59]$ for the case $k_{c} \neq 0$ and in [55] for the case $k_{c}=0$. In order to explain the ideas we avoid several technical details by assuming for this proof that $\left.D^{2} f\right|_{u=0}=0$.

In order to prove the long-time existence the variable $w=\epsilon^{-1} u$ is introduced. Then $w$ satisfies

$$
\partial w=\mathcal{A} w+h_{0}(w),
$$

with

$$
\left.w\right|_{t=0}=w_{0}, \quad\left\|w_{0}\right\|_{C_{b}^{0}} \leq C_{1}, \quad \text { and } \quad\left\|h_{0}(w)\right\|_{C_{b}^{0}} \leq C \epsilon^{2}\|w\|_{C_{b}^{0}}^{3}
$$

for all $\varepsilon\|w\|_{C_{b}^{0}} \leq M$ for a fixed $M>0$. With the variation of constant formula and Gronwall's inequality the existence of a $T_{0}>0$ and an $\epsilon_{0}>0$ follows such that for all $\epsilon \in\left(0, \epsilon_{0}\right)$ there is an $\mathcal{O}(1)$-bounded solution $w \in C\left(\left[0, T_{0} / \epsilon^{2}\right], C_{b}^{0}\right)$ with $\left.w\right|_{t=0}=w_{0}$. Equivalent to the assertion of the theorem is to show the $\mathcal{O}(1)$-boundedness of

$$
\|w\|_{C_{b}^{n, \epsilon}}=\sum_{j=0}^{n}\left\|\epsilon^{-j} \partial_{x}^{j} w\right\|_{C_{b}^{0}}
$$

for $t=T_{0} / \epsilon^{2}$. Since $\|\cdot\|_{C_{b}^{0}}=\|\cdot\|_{C_{b}^{0, \epsilon}}$ the variation of constant formula yields

$$
\begin{aligned}
\left\|\left.w\right|_{t=T_{0} / \epsilon^{2}}\right\|_{C_{b}^{1, \epsilon}} & \leq\left\|\mathrm{e}^{\mathcal{A} T_{0} / \epsilon^{2}}\right\|_{C_{b}^{0, \epsilon} \rightarrow C_{b}^{1, \epsilon}}\left\|\left.w\right|_{t=0}\right\|_{C_{b}^{0, \epsilon}}+\int_{0}^{T_{0} / \epsilon^{2}}\left\|\mathrm{e}^{\mathcal{A}\left(T_{0} / \epsilon^{2}-s\right)}\right\|_{C_{b}^{0, \epsilon} \rightarrow C_{b}^{1, \epsilon}} d s C \epsilon^{2} \sup _{s \in\left[0, T_{0} / \epsilon^{2}\right]}\|w(s)\|_{C_{b}^{0}}^{3} \\
& \leq C\left\|\left.w\right|_{t=0}\right\|_{C_{b}^{0, \epsilon}}+C \epsilon^{2} \int_{0}^{T_{0} / \epsilon^{2}} \max \left(1, \epsilon^{-1}\left(T_{0} / \epsilon^{2}-s\right)^{-1 / 2}\right) d s=\mathcal{O}(1)
\end{aligned}
$$

due to (11). We start (7) again, but now with $\left\|\left.w\right|_{t=0}\right\|_{C_{b}^{1, \epsilon}}=\mathcal{O}(1)$. The nonlinear terms satisfy

$$
\left\|h_{0}(w)\right\|_{C_{b}^{1, \epsilon}} \leq C \epsilon^{2}\|w\|_{C_{b}^{1, \epsilon}}^{3} .
$$

With the variation of constant formula and Gronwall's inequality the existence of a $T_{1}>0$ and an $\epsilon_{0}>0$ follows such that for all $\epsilon \in\left(0, \epsilon_{0}\right)$ there is an $\mathcal{O}(1)$-bounded solution $w \in C\left(\left[0, T_{1} / \epsilon^{2}\right], C_{b}^{1, \epsilon}\right)$ with $\left.w\right|_{t=0}=w_{0}$. The variation of constant formula yields

$$
\begin{aligned}
\left\|\left.w\right|_{t=T_{1} / \epsilon^{2}}\right\|_{C_{b}^{2, \epsilon}} & \leq\left\|\mathrm{e}^{\mathcal{A} T_{1} / \epsilon^{2}}\right\|_{C_{b}^{1, \epsilon} \rightarrow C_{b}^{2, \epsilon}}\left\|\left.w\right|_{t=0}\right\|_{C_{b}^{1, \epsilon}}+\int_{0}^{T_{1} / \epsilon^{2}}\left\|\mathrm{e}^{\mathcal{A}\left(T_{1} / \epsilon^{2}-s\right)}\right\|_{C_{b}^{1, \epsilon} \rightarrow C_{b}^{2, \epsilon}} d s C \epsilon^{2} \sup _{s \in\left[0, T_{1} / \epsilon^{2}\right]}\|w(s)\|_{C_{b}^{1, \epsilon}}^{3} \\
& \leq C\left\|\left.w\right|_{t=0}\right\|_{C_{b}^{1, \epsilon}}+C \epsilon^{2} \int_{0}^{T_{1} / \epsilon^{2}} \max \left(1, \epsilon^{-1}\left(T_{1} / \epsilon^{2}-s\right)^{-1 / 2}\right) d s=\mathcal{O}(1)
\end{aligned}
$$


Since this scheme can be iterated and since

$$
\begin{aligned}
\left\|\left.P_{2} w\right|_{t=T_{1} / \epsilon^{2}}\right\|_{C_{b}^{0}} & \leq\left\|P_{2} \mathrm{e}^{\mathcal{A} T_{1} / \epsilon^{2}}\right\|_{C_{b}^{0} \rightarrow C_{b}^{0}}\left\|\left.w\right|_{t=0}\right\|_{C_{b}^{0}}+\int_{0}^{T_{1} / \epsilon^{2}}\left\|P_{2} \mathrm{e}^{\mathcal{A}\left(T_{1} / \epsilon^{2}-s\right)}\right\|_{C_{b}^{0} \rightarrow C_{b}^{0}} d s C \epsilon^{2} \sup _{s \in\left[0, T_{1} / \epsilon^{2}\right]}\|w(s)\|_{C_{b}^{0}}^{3} \\
& \leq C \mathrm{e}^{-\beta T_{1} / \epsilon^{2}}\left\|\left.w\right|_{t=0}\right\|_{C_{b}^{1, \epsilon}}+C \epsilon^{2} \int_{0}^{T_{1} / \epsilon^{2}} \mathrm{e}^{-\beta\left(T_{1} / \epsilon^{2}-s\right)} d s=\mathcal{O}\left(\epsilon^{2}\right)
\end{aligned}
$$

the result follows for all $n \in \mathbb{N}$.

\subsection{Proof of Theorem 3}

The residual

$$
\operatorname{Res}_{1}(u)=-\partial_{t} u_{1}+\epsilon^{2} u_{1}+\partial_{x}^{2} u_{1}+N_{1}(u), \quad \operatorname{Res}_{2}(u)=-\partial_{t} v+Q v+\partial_{x}^{2} v+N_{2}(u)
$$

contains the information how much a function $u$ fails to satisfy system (7). As in [47] it easily follows that

$$
\sup _{t \in\left[0, T_{1} / \epsilon^{2}\right]}\left\|\operatorname{Res}_{1}\left(\psi_{\epsilon}\left(A\left(\epsilon^{2} t\right)\right)\right)\right\| \leq C_{\operatorname{Res}} \epsilon^{4} \quad \text { and } \sup _{t \in\left[0, T_{1} / \epsilon^{2}\right]}\left\|\operatorname{Res}_{2}\left(\psi_{\epsilon}\left(A\left(\epsilon^{2} t\right)\right)\right)\right\| \leq C_{\operatorname{Res}} \epsilon^{3} .
$$

The error $R=\left(R_{1}, R_{2}\right)$ with

$$
\epsilon^{2} R_{1}=u_{1}-\left\langle e_{1}, \psi_{\epsilon}(A)\right\rangle_{\mathbb{R}^{d}} \quad \text { and } \quad \epsilon^{3} R_{2}=v-P_{2} \psi_{\epsilon}(A)
$$

satisfies

$$
\partial_{t} R_{1}=\epsilon^{2} R_{1}+\partial_{x}^{2} R_{1}+g_{1}(u), \quad \partial_{t} R_{2}=Q R_{2}+\partial_{x}^{2} R_{2}+g_{2}(u)
$$

where

$$
\left\|g_{1}\right\|_{C_{b}^{0}} \leq C \epsilon^{2}\left\|R_{1}\right\|_{C_{b}^{0}}+C \epsilon^{2}\left\|R_{2}\right\|_{C_{b}^{0}}+C \epsilon^{3}\|R\|_{C_{b}^{0}}^{2}+C_{\text {Res }} \epsilon^{2}, \quad\left\|g_{2}\right\|_{C_{b}^{0}} \leq C\left\|R_{1}\right\|_{C_{b}^{0}}+C \epsilon\left\|R_{2}\right\|_{C_{b}^{0}}+C \epsilon\|R\|_{C_{b}^{0}}^{2}+C_{\text {Res }},
$$

if $\|\epsilon R\|_{C_{b}^{0}} \leq M$ for a fixed $M$. Applying the variation of constant formula to these equations and making the abbreviations $S_{i}(t):=\sup _{s \leq t}\left\|R_{i}(s)\right\|_{C_{b}^{0}}$ allows us to obtain the estimates

$$
\begin{aligned}
& S_{1}(t) \leq \varepsilon^{2} C \int_{0}^{t}\left(S_{1}(\tau)+S_{2}(\tau)+\varepsilon\left(S_{1}(\tau)+S_{2}(\tau)\right)^{2}\right) d \tau+C\left(S_{1}(0)+C_{\mathrm{Res}}\right) \leq \varepsilon^{2} C_{3} \int_{0}^{t}\left(S_{1}(\tau)+S_{2}(\tau)\right) d \tau+C_{4}, \\
& S_{2}(t) \leq C\left(S_{1}(t)+\varepsilon S_{2}(t)+\varepsilon\left(S_{1}(t)+S_{2}(t)\right)^{2}\right)+C\left(S_{2}(0)+C_{\mathrm{Res}}\right) \leq C_{5} S_{1}(t)+C_{6},
\end{aligned}
$$

where $C_{4}=C\left(S_{1}(0)+C_{\text {Res }}\right)+1, C \varepsilon T_{1}\left(C_{c}+C_{s}\right)^{2}<1, C_{6}=C\left(S_{2}(0)+C_{\text {Res }}\right)+1$, and $C\left(\varepsilon C_{s}+\varepsilon\left(C_{c}+C_{s}\right)^{2}\right)<1$ as long as $S_{1} \leq C_{c}$ and $S_{2} \leq C_{s}$ for two constants $C_{c}$ and $C_{s}$ to be defined later. Inserting the second inequality in the first one gives the estimate

$$
S_{1}(t) \leq C_{4}+C_{6} T_{1}+\varepsilon^{2} C_{3} \int_{0}^{t}\left(1+C_{5}\right) S_{1}(\tau) d \tau
$$

Applying Gronwall's inequality to this gives $S_{1}(t) \leq\left(C_{4}+C_{6} T_{1}\right) \mathrm{e}^{C_{3}\left(1+C_{5}\right) T_{1}}=: C_{c}$. In the second equation we obtain by this $S_{2}(t) \leq C_{5} C_{c}+C_{6}=: C_{s}$. Choosing now $\varepsilon_{0}>0$ in such a way that the restrictions on $\varepsilon$ are fulfilled we are done.

\subsection{Proof of Theorem 7}

In order to construct these solutions the stationary system of (7) is written as a first order system

$$
\partial_{x} u_{j}=v_{j}, \quad \partial_{x} v_{j}=-f_{j}\left(\alpha, u_{1}, \ldots, u_{d}\right)
$$

with $x$ as new time variable. For $\alpha=0$ the eigenvalues $\mu$ of the linearisation of this spatial dynamics formulation satisfy

$$
\mu u_{j}=v_{j}, \mu\left(v_{1}, \ldots, v_{d}\right)=-\left.D f\right|_{u=0} u
$$

and so $\left.D f\right|_{u=0} u=-\mu^{2} u$. Since $\left.D f\right|_{u=0} \varphi_{j}=\lambda(0, j) \varphi_{j}$ the relation $\lambda(0, j)=-\mu_{j}^{2}$ between the temporal eigenvalues $\lambda(0, j)$ and the spatial eigenvalues $\mu$ follow. Therefore, there are two eigenvalues $\mu=0$ (a Jordan-block), and the rest of the eigenvalues $\mu_{j}$ is bounded away from the imaginary axis. Thus, the assumptions of the center manifold theorem are satisfied and a two-dimensional center manifold can be constructed. By making the ansatz $u(x)=\epsilon A(\epsilon x) \varphi_{1}+\mathcal{O}\left(\epsilon^{2}\right)$ it turns out that the vector field on the center manifold is given in lowest order by

$$
\partial_{X} A=A_{1}, \quad \partial_{X} A_{1}=-A+A^{3}+\mathcal{O}(\epsilon) .
$$

For $\epsilon=0$ this system is the stationary Allen-Cahn equation which possesses a heteroclinic connection between $\left(A, A_{1}\right)=(1,0)$ and $(-1,0)$. Since $(7)$ is reflection symmetric $(x \mapsto-x, u \mapsto u)$ this heteroclinic connection persists under higher order perturbations, i.e. for $(7)$.

Stability follows by looking at the linearisation around $\tilde{I}_{1}$. The continuous spectrum of this linearisation lies strictly in the left half plane. Since (7) can be approximated by (12) the same is true for the linearised systems, and so there exists a one to one correspondence between the discrete eigenvalues of both equations. All eigenvalues of (12) are only perturbed a little bit and stay for $\epsilon>0$ sufficiently small strictly in the left half plane, except of the zero eigenvalue from which we already know that it remains in zero with associated eigenvector $\partial_{x} U$. The nonlinear stability again follows with the help of the center manifold theorem (see $[26])$. 


\section{Acknowledgements}

The author is grateful for discussions with J. RougEMENT. The research was partially supported by Deutsche Forschungsgemeinschaft under the grant Mi 459/2-3.

\section{References}

1 Afendikov, A.; KirchgÄssner, K.: Spatial dynamics approach to Kolmogorov flows on plane torus. ZAMM 78 (1998) Suppl. 1, S211-S212.

2 Afendikov, A.; Mielke, A.: On the unfolding of reversible vector fields with SO(2)-symmetry and a non-semisimple eigenvalue 0. Doklady Mat. 60 (1999), 370-402.

3 Afendikov, A.; Mielke, A.: Multi-pulse solutions to the Navier-Stokes problem between parallel plates. Z. Angew. Math. Phys. (to appear).

4 Bollerman, P.: Validity of the Ginzburg-Landau approximation in 2-dimensional Poiseuille flow. In: Mielke, A. et al. (eds.): Structure and dynamics of nonlinear waves in fluids. Proc. IUTAM/ISIMM symposium, Hannover, Germany, August 17-20, 1994. World Scientific Adv. Ser. Nonlinear Dyn. 7, Singapore 1995, pp. 158-164.

5 Bollerman, P.; van Harten, A.; Schneider, G.: On the justification of the Ginzburg-Landau approximation. In: Doelman, A. et al. (eds.): Nonlinear dynamics and pattern formation in the natural environment. Proceedings of the international conference held in Noordwijkerhout, The Netherlands, July 4-7, 1994. Harlow: Longman. Pitman Res. Notes Math. Ser. 335 (1995), pp. $20-36$.

6 Bricmont, J.; Kupiainen, A.: Renormalization group and the Ginzburg-Landau equation. Comm. Math. Phys. 150 (1992), $193-208$.

7 Bricmont, J.; Kupiainen, A.: Stability of moving fronts in the Ginzburg-Landau equation. Comm. Math. Phys. 159 (1994), $287-318$.

8 Shui-Nee Chow; Hale, J. K.: Methods of bifurcation theory. Grundlehren der Mathematischen Wissenschaften, Bd. 251. Springer-Verlag, New York-Berlin-Heidelberg 1982.

9 Coddington, E. A.; Levinson, N.: Theory of ordinary differential equations. McGraw-Hill Book Company, Inc., 1955.

10 Collet, P.; Eckmann, J.-P.: The existence of dendritic fronts. Comm. Math. Phys. 107 (1986), $39-92$.

11 Collet, P.; Eckmann, J.-P.: The time dependent amplitude equation for the Swift-Hohenberg problem. Comm. Math. Phys. 132 (1990), 139-153.

12 Collet, P.; Eckmann, J.-P.; Epstein, H.: Diffusive repair for the Ginsburg-Landau equation. Helv. Phys. Acta 65 (1992), $56-92$.

13 Di Prima, R.C.; Eckhaus, W.; Segel, L. A.: Nonlinear wave-number interaction in near-critical two-dimensional flows. J. Fluid Mech. 49 (1971), 705-744.

14 Doelman, A.: Finite-dimensional models of the Ginzburg-Landau equation. Nonlinearity 4 (1991), $231-250$.

15 Eckhaus, W.: The Ginzburg-Landau equation is an attractor. J. Nonlinear Sci. 3 (1993), 329-348.

16 Eckmann, J.-P., Wayne, C. E.: Propagating fronts and the center manifold theorem. Comm. Math. Phys 136 (1991), $285-307$.

17 Eckmann, J.-P.; Wayne, C. E.: The non-linear stability of front solutions for parabolic partial differential equations. Comm. Math. Phys. 161 (1994), 323-334.

18 Eckmann, J.-P.; Wayne, C. E.; Wittwer, P.: Geometric stability analysis of periodic solutions of the Swift-Hohenberg equation. Comm. Math. Phys. 190 (1997), 173-211.

19 Fisher, R.: The advance of advantageous genes. Ann. Eugenics 7 (1937), 355-369.

20 Gallay, T.: Local stability of critical fronts in nonlinear parabolic partial differential equations. Nonlinearity 7 (1994), $741-764$.

21 Gallay, T.; Mielke, A.: Diffusive mixing of stable states in the Ginzburg-Landau equation. Comm. Math. Phys. 199 (1998), $71-97$.

22 Golubitsky, M.; Schaeffer, D. G.: Singularities and groups in bifurcation theory. Vol. I. Appl. Math. Sci. 51. Springer-Verlag, New York etc. 1985 .

23 Golubitsky, M.; Stewhrt, I.; Schaeffer, D. G.: Singularities and groups in bifurcation theory. Vol. II. Appl. Math. Sci. 69. Springer-Verlag, New York etc. 1988.

24 Guckenheimer, J.; Holmes, P.: Nonlinear oscillations, dynamical systems and bifurcations of vector fields. Appl. Math. Sci. 42. Springer, New York 1983.

25 Haragus, M.; Schneider, G.: Bifurcating fronts for the Taylor-Couette problem in infinite cylinders. Z. Angew. Math. Phys. 50 (1999), 120-151.

26 Henry, D.: Geometric theory of semilinear parabolic equations. Lecture Notes Math. 840. Springer 1981.

27 Iooss, G.; Mielke, A., Demay, Y.: Theory of steady Ginzburg-Landau equation in hydrodynamic stability problems. Europ. J. Mech., B/Fluids 8 (1989), 229-268.

28 Iooss, G.; Mielke, A.: Bifurcating time-periodic solutions of Navier-Stokes equations in infinite cylinders. J. Nonlinear Sci. 1 (1991), 107-146.

29 Iooss, G.; Kirchgässner, K.: Water waves for small surface tension: an approach via normal form. Proc. Roy. Soc. Edinb. 122A (1992), 267-299.

30 Kapitula, T.: On the nonlinear stability of plane waves for the Ginzburg-Landau equation. Comm. Pure. Appl. Math. 47 (1994), $831-841$.

31 Kapitula, T.; Maier-PaApe, S.: Spatial dynamics of time periodic solutions for the Ginzburg-Landau equation. Z. Angew. Math. Phys. 47 (1996), 265-305.

32 Kapitula, T.; SAndstede, B.: Stability of bright solitary-wave solutions to perturbed nonlinear Schrödinger equations. Physica D 124 (1998), 58-103.

33 KirchGässner, K.: Wave solutions of reversible systems and applications. J. Diff. Equ. 45 (1982), $113-127$.

34 Kirrmann, P.; Schneider, G., Mielke, A.: The validity of modulation equations for extended systems with cubic nonlinearities. Proc. Roy. Soc. Edinb., Sect. A 122 (1992), 85-91.

35 Knobloch, E.: Nonlocal amplitude equations. In: KaI, S. (ed.): Pattern formation in complex dissipative systems. World Scientific 1992 , pp. $263-274$.

36 Kolmogorov, A. N.; Petrovskit, I. G.; Piskunov, N. S.: Etude de la diffusion avec croissance de la qualité de matière et son applications à un problème biologique. Moscow Univ. Math. Bull. 1 (1937), 1-25. 
37 Kuramoto, Y.: Chemical oscillations, waves, and turbulence. Springer, Berlin 1984.

38 Marsden, J. E.; McCracken, M.: The Hopf bifurcation and its applications. Appl. Math. Sci. 19. Springer-Verlag, New YorkHeidelberg-Berlin 1976.

39 Melbourne, I.: Derivation of the time-dependent Ginzburg-Landau equation on the line. J. Nonlinear Sci. 8 (1998), 1-15.

40 Melbourne, I.: Steady-state bifurcation with Euclidean symmetry. Trans. Am. Math. Soc. 351 (1999), 1575-1603.

41 Mielke, A.: Saint Venant's problem and semi-inverse solutions in nonlinear elasticity. Arch. Rat. Mech. Anal. 102 (1988), $205-229$.

42 Mielke, A., Schneider, G.: Attractors for modulation equations on unbounded domains - existence and comparison -. Nonlinearity 8 (1995), 743-768.

43 Mielke, A.: The complex Ginzburg-Landau equation on large and unbounded domains: Sharper bounds and attractors. Nonlinearity 10 (1997), 199-222.

44 Mielke, A., The Ginzburg-Landau equation in its role as a modulation equation. In: Fiedler, B. et al. (eds.): Handbook of dynamical systems. III. Towards applications. Springer-Verlag (to appear).

45 Newell, A.; Whitehead, J.: Finite bandwidth, finite amplitude convection. J. Fluid Mech. 38 (1969), 279-303.

46 Rougement, J.: Dynamics of kinks in the Ginzburg-Landau equation: approach to a metastable shape and collapse of embedded pairs of kinks. Nonlinearity 12 (1999), 539-554.

47 Schneider, G.: A new estimate for the Ginzburg-Landau approximation on the real axis. J. Nonlinear Sci. 4 (1994), $23-34$.

48 Schneider, G.: Error estimates for the Ginzburg-Landau approximation. Z. Angew. Math. Phys. 45 (1994), $433-457$.

49 Schneider, G.: Global existence via Ginzburg-Landau formalism and pseudo-orbits of Ginzburg-Landau approximations. Comm. Math. Phys. 164 (1994), 157-179.

50 Schneider, G.: Justification of modulation equations in domains with $n \geq 2$ unbounded space directions. In: Mielke, A. et al. (eds.): Structure and dynamics of nonlinear waves in fluids. Proc. IUTAM/ISIMM symposium, Hannover, Germany, August 17-20, 1994. World Scientific. Adv. Ser. Nonlinear Dyn. 7, Singapore 1995, pp. 383-391.

51 Schneider, G.: Analyticity of Ginzburg-Landau modes. J. Diff. Equ. 121 (1995), 233-257.

52 Schneider, G.: Validity and limitation of the Newell-Whitehead equation. Math. Nachr. 176 (1995), 249-263.

53 Schneider, G.: Diffusive stability of spatial periodic solutions of the Swift-Hohenberg equation. Comm. Math. Phys. 178 (1996), $679-702$.

54 Schneider, G.: Justification of mean-field coupled modulation equations. Proc. Roy. Soc. Edinb., Sect. A 127 (1997), 639-650.

55 Schneider, G.: Hopf-bifurcation in spatially extended reaction-diffusion systems. J. Nonlinear Sci. 8 (1998), $17-41$.

56 Schneider, G.: Müller, J. Bifurcation analysis for a spatially extended random walk system in mathematical biology. Comm. Appl. Analysis (to appear).

57 Schneider, G.: Aspects of modulational dynamics - Diffusive versus pulse behavior -. Proc. STAMM 98. Nice May 25-29, 1998.

58 Schneider, G.: Nonlinear stability of Taylor-vortices in infinite cylinders. Arch. Rat. Mech. Anal. 144 (1998), 121-200.

59 Schneider, G.: Global existence results for pattern forming processes in infinite cylindrical domains - Applications to 3D NavierStokes problems -. J. Math. Pur. Appl. 78 (1999), 265-312.

60 SChneider, G.: Cahn-Hilliard description of secondary flows of a viscous incompressible fluid in an unbounded domain. ZAMM 79 (1999), 615-626.

61 Takac, P.; Bollerman, P.; Doelman, A.; van Harten, A.; Titi, E.S.: Analyticity of essentially bounded solutions to semilinear parabolic systems and validity of the Ginzburg-Landau equation. SIAM J. Math. Anal. 27 (1996), 424-448.

62 Shepeleva, A.: On the validity of the degenerate Ginzburg-Landau equation. Math. Methods Appl. Sci. 20 (1997), $1239-1256$.

63 Spektrum der Wissenschaft, Software Dossier 2/1999. Spektrum der Wissenschaft Verlagsgesellschaft, Heidelberg 1999.

64 Vanderbaumhede, A.: Centre manifolds, normal forms and elementary bifurcations. Dynamics Reported 2 (1989), 89-169.

65 Vanderbauwhede, A.; Iooss, G.: Centre manifold theory in infinite dimensions. Dynamics Reported, N. S. 1 (1992), $125-163$.

66 van Harten, A.: On the validity of Ginzburg-Landau's equation. J. Nonlinear Sci. 1 (1991), 397-422.

Received August 25, 1999, revised August 16, 2000, accepted August 23, 2000

Address: Prof. Guido SchneIDER, Mathematisches Institut, Universität Bayreuth, D-95440 Bayreuth, Germany, email: guido.schneider@uni-bayreuth.de 